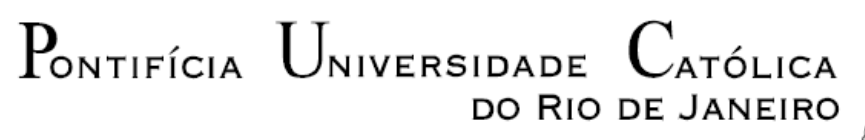

DO RIO DE JANEIRO

Rodrigo Antônio Farinazzo

\title{
A DEMANDA POR ENERGIA ELÉTRICA RESIDENCIAL NO BRASIL: 2011-2020
}

Dissertação de Mestrado

Dissertação apresentada como requisito parcial para obtenção do grau de Mestre pelo Programa de PósGraduação em Engenharia Elétrica da PUC-Rio.

Orientador: Prof. Reinaldo Castro Souza Co-Orientador: Prof. José Francisco Moreira Pessanha 


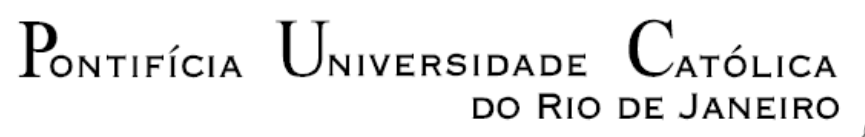

DO RIO DE JANEIRO

Rodrigo Antônio Farinazzo

\section{A DEMANDA POR ENERGIA ELÉTRICA RESIDENCIAL NO \\ BRASIL: 2011-2020}

Dissertação apresentada como requisito parcial para obtenção do título de Mestre pelo Programa de PósGraduação em Engenharia Elétrica da PUC-Rio. Aprovada pela Comissão Examinadora abaixo assinada.

Prof. Reinaldo Castro Souza

Orientador

Departamento de Engenharia Elétrica - PUC-Rio

Prof. José Francisco Moreira Pessanha

Co-Orientador

UERJ

Prof. Alexandre Zanini

UFJF

Prof. André Frossard Pereira de Lucena

UFRJ

Prof. José Eugênio Leal

Coordenador Setorial do Centro Técnico-Científico

Rio de Janeiro, 10 de Maio de 2012. 
Todos os direitos reservados. É proibida a reprodução total ou parcial do trabalho sem autorização da Universidade, do autor e do orientador.

Rodrigo Antônio Farinazzo

Graduou-se em Ciências Econômicas pela Universidade Federal de Juiz de Fora em 2009. Especialista em Métodos de Apoio à Decisão pela mesma instituição, em 2011.

Ficha Catalográfica

Farinazzo, Rodrigo Antônio

A Demanda por Energia Elétrica Residencial no Brasil: 2010-2020 / Rodrigo Antônio Farinazzo; orientador Reinaldo Castro Souza; co-orientador: José Francisco Moreira Pessanha. - 2012.

89f. : il. (color); $30 \mathrm{~cm}$

Dissertação (mestrado) - Pontifícia Universidade Católica do Rio de Janeiro, Departamento de Engenharia Elétrica, 2012.

Inclui bibliografia

1. Engenharia Elétrica - Teses. 2. Cointegração. 3. Taxa de Chefia. 4. Decomposição do Índice de Divisia de Média Logarítimica I (LMDI I) 5. Previsões. I. Souza, Reinaldo Castro. II. Pessanha, José Francisco Moreira. III. Pontifícia Universidade Católica do Rio de Janeiro. Departamento de Engenharia Elétrica. IV. Título. 
À minha família, sempre presente.

À minha amada noiva, por toda dedicação, incentivo e carinho. 


\section{Agradecimentos}

Agradeço a Deus pelas oportunidades que me foram dadas na vida e por ter iluminado o meu caminho diante de mais este desafio.

A minha amada noiva Paula Coelho Araujo, grande companheira de caminhada, pelo amor, paciência, carinho, compreensão e, principalmente, por estar sempre ao meu lado me incentivando.

Aos meus pais Ana Maria de Lima e Antônio Laerte Farinazzo, assim como a minha irmã Nívea Maria Farinazzo, por me incentivarem, confiarem em mim e também, por todo apoio financeiro.

Ao CAPES e à PUC-Rio, pelos auxílios concedidos, sem os quais este trabalho não poderia ter sido realizado.

Agradeço também ao meu orientador e professor Reinaldo Castro Souza, pela confiança depositada em mim desde o início desta jornada e aos seus colaboradores Ana Paiva e Flávio Nascimento, por suas palavras amigas, incentivadoras e confortantes nos momentos difíceis.

A todo corpo docente ligado ao Departamento de Engenharia Elétrica da Pontifícia Universidade Católica do Rio de Janeiro (PUC-RJ) meus sinceros agradecimentos por todo o conhecimento transmitido e dedicação.

Aos meus grandes amigos, Carlos Chang, Erika Victorina, Flávia Fontanet, Fernando Cyrino, Gilson Gonçalves, Gláucia Estefânia, Guilherme Armando, João Paulo e Rafael Morais obrigado por terem me ajudado a enxergar de uma forma mais fácil e clara as dificuldades, pelas horas despendidas nos grupos de estudo e de trabalho, além das conversas descontraídas.

Em especial, gostaria de agradecer ao meu amigo e co-orientador professor José Francisco Moreira Pessanha por ter dedicado parte de seu precioso tempo nas discussões frutíferas que deram origem a este estudo, bem como por sua dedicação, atenção e incentivo no sentido de não me deixar desistir desta empreitada, demonstrando, assim, o verdadeiro sentido da amizade.

Muito obrigado a Todos que contribuíram de maneira direta ou indireta tornando este sonho uma realidade! 


\section{Resumo}

Farinazzo, Rodrigo Antônio; Souza, Reinaldo Castro; Pessanha, José Francisco Moreira. A Demanda por Energia Elétrica Residencial no Brasil: 2011-2020. Rio de Janeiro, 2012, 89p. Dissertação de Mestrado Departamento de Engenharia Elétrica, Pontifícia Universidade Católica do Rio de Janeiro.

Com este trabalho, objetivou-se quantificar as relações entre a demanda de energia elétrica e alguns de seus determinantes na classe Residencial do Brasil. Inicialmente, é feita uma breve discussão sobre o consumo residencial de energia no País ao longo das quatro últimas décadas, a fim de se conhecer o mesmo dentro de um contexto mais amplo. Posteriormente, adotou-se uma modelagem econométrica objetivando estimar as elasticidades-preço, renda e preço de eletrodomésticos. Após identificar que as séries temporais das variáveis estudadas são não estacionárias, optou-se pela utilização do conceito de Cointegração. Em seguida, foram estimados os Modelos de Correção de Erros Vetoriais (VECM) os quais foram utilizados para projetar o consumo de energia elétrica para o período 2011-2020. Num terceiro momento, entendendo o crescimento populacional e o número de domicílios como fatores importantes na determinação do número de unidades consumidoras residenciais e, consequentemente, na demanda de energia elétrica, foi adotado o método Taxa de Chefia baseado no modelo idade-períodocoorte (IPC) objetivando projetar a Taxa de Chefia e o número de domicílios que deverão ser atendidos pelas concessionárias de distribuição durante o período 2011-2020. Por fim, diante das informações obtidas junto às referidas projeções, aplicou-se o método de decomposição do índice de Divisia de média logarítmica I (LMDI I) a fim de explicar a variação da demanda de energia sob a ótica de três variáveis, aqui denominadas Consumo Médio, Taxa de Atendimento e Domicílio.

\section{Palavras-chave}

Demanda de Energia Elétrica; Previsões de Longo Prazo; Classe Residencial; Cointegração; Números de Domicílios, Taxa de Chefia; Índice de Divisia. 


\section{Abstract}

Farinazzo, Rodrigo Antônio; Souza, Reinaldo Castro (Advisor); Pessanha, José Francisco Moreira (Co-Advisor). The Demand for Residential Electricity in Brazil: 2011-2020. Rio de Janeiro, 2012. 89p. MSc. Dissertation - Departamento de Engenharia Elétrica, Pontifícia Universidade Católica do Rio de Janeiro.

This work aims to quantify the relations between the electricity demand and some of its determinants in the Residential sector of Brazil. To begin with a short discussion is carried out on the Residential energy consumption in the country throughout the last four decades so as to get to know the residential consumption within a wider context. After, we adopted an econometric modeling aiming to estimate price elasticities, income and price of home appliances. As the series of the variables studied were proved non-stationary, the Cointegration approach was adopted. Vector Error Correction Models (VECM) were estimated and used to project the consumption of electric energy during the 2011-2020 period. The third point concerns, understanding the population growth and the number of households as important factors in determining the number of residential consumer units and, consequently, the demand for electricity, has been adopted the method leadership rate based on age-period-cohort model (APC) aiming to projecting the rate at the leadership rate and the number of homes that should be attended by energy concession during the period 2010-2020. Finally, in face of information obtained from these forcasts, we applied the logarithmic mean Divisia index decomposition method I (LMDI I) in order to explain the variation in energy demand on the optics of the three variables, called Average Consumption, Attendance rate and Household.

\section{Keywords}

Electricity Demand; Long-Term Forecast; Residential Electricity; Cointegration; Number of Homes; Leadership Rate; Divisia Index. 


\section{Sumário}

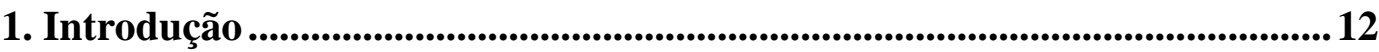

1.1. Considerações Gerais para o caso brasileiro ............................................ 12

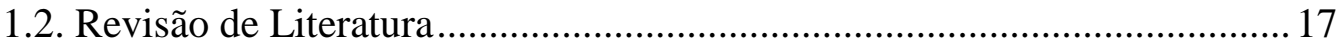

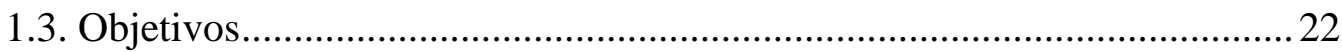

1.4. Organização da Dissertação ...................................................................... 23

2. Metodologias para Projeção do Mercado da Classe Residencial .................. 24

2.1. Modelagem Econométrica ....................................................................... 25

2.1.1. Testes de Raízes Unitárias ........................................................28

2.1.2. Análise de cointegração ............................................................ 32

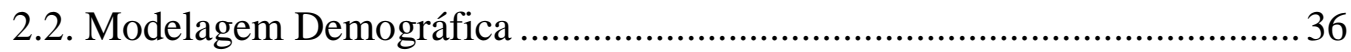

2.2.1. Projeção do número de domicílios ocupados...................................38

2.2.2. Projeção do número de domicílios de uso ocasional .........................47

2.3. Método Índice de Divisia de Média Logarítmica I (LMDI I) .................... 48

3. Análise e Discussão dos Resultados.........................................................................52

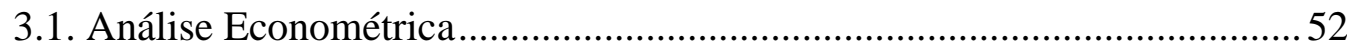

3.1.1. Especificação Empírica.............................................................. 52

3.1.2. Estimativas da Elasticidade-Preço e da Elasticidade-Renda...............54

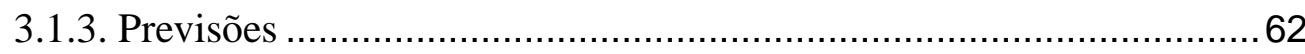

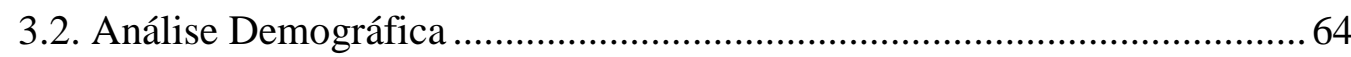

3.2.1. Projeções da Taxa de Chefia por faixa etária e sexo ......................... 64

3.2.2. Projeção da Taxa de Chefia da população masculina e feminina .......66

3.2.3. Projeção do número de domicílios .................................................... 71

3.2.4. Projeção do número de unidades consumidoras da classe

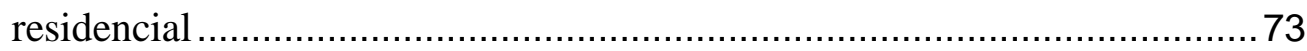

3.3. Método do Índice de Divisia de Média Logarítmica I (LMDI I) ............... 73

4. Conclusões e Considerações Finais ...............................................................79

5. Referências Bibliográficas ...............................................................................82 


\section{Lista de figuras}

Figura 1: Evolução do consumo residencial de energia elétrica $(\mathrm{GWh})$ e da taxa média anual de variação do consumo residencial (\%), Brasil: 1980-2010 ..........13

Figura 2: Consumo Nacional de Energia Elétrica na Rede por Classe .......................14

Figura 3: Evolução do Consumo de Energia Elétrica por Unidade Consumidora (1980 a 2010) .15

Figura 4: Evolução da renda domiciliar per capita anual, Brasil: 1980-2009

(em R\$ [2009]) 16

Figura 5: Pirâmides etárias da população brasileira em 2010 e 2020 (IBGE) ............40

Figura 6: Seqüência de coortes transversais .41

Figura 7: Razão do número de domicílios de uso ocasional pelo número de domicílios ocupados

Figura 8: Métodos derivados do índice de Divisia úteis na análise de decomposição do uso da energia .49

Figura 9: Dinâmica da taxa de Chefia nas populações masculina e feminina ..... .66

Figura 10: Comportamento da Taxa de Chefia da população masculina....................68

Figura 11: Comportamento da Taxa de Chefia da população feminina......................69

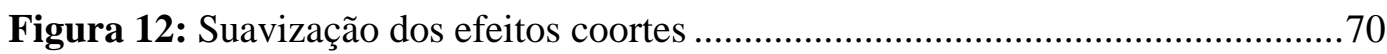

Figura 13: Projeção da Taxa de Chefia para a população masculina no ano de 2020 .71

Figura 14: Projeção da Taxa de Chefia para a população feminina no ano de 2020 .71

Figura 15: Decomposição das variações do consumo residencial de energia elétrica, Brasil: 1980-2020 (GW) .74

Figura 16: Agregação temporal dos Efeitos Consumo Médio, Taxa de Atendimento e Domicílio. 


\section{Lista de tabelas}

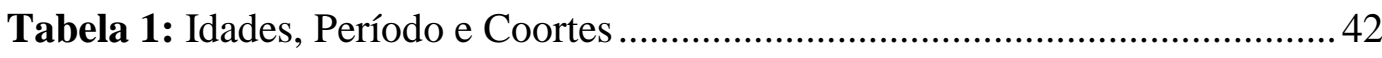

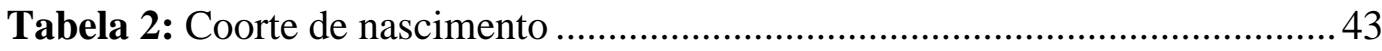

Tabela 3: Taxa de chefia da população masculina .............................................. 43

Tabela 4: Taxa de chefia da população feminina ................................................ 43

Tabela 5: Composição dos efeitos na formação do logito da taxa de chefia......... 46

Tabela 6: Teste de Raiz Unitária das Variáveis..................................................... 55

Tabela 7: Estrutura de Defasagem do modelo VAR (p).....................................55

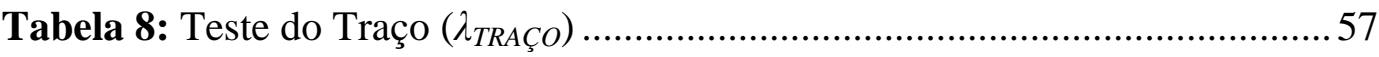

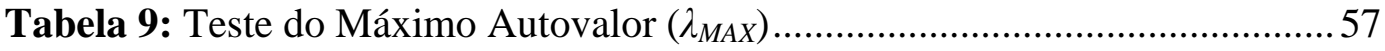

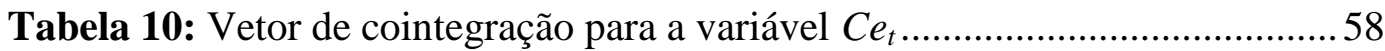

Tabela 11: Elasticidade Preço e Renda para a Classe Residencial.......................59

Tabela 12: Estimação do Modelo VEC referente à variável $C e_{t} \ldots \ldots \ldots \ldots \ldots \ldots \ldots \ldots . . . . . . . . . . . . .61$

Tabela 13: Projeções para o Consumo de Energia Elétrica................................... 63

Tabela 14: Taxa de Chefia por idade, período e coorte da população

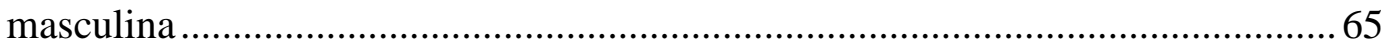

Tabela 15: Taxa de Chefia por idade, período e coorte da população feminina 65

Tabela 16: Estimativas dos Efeitos idade-período-coorte para a população masculina.

Tabela 17: Estimativas dos Efeitos idade-período-coorte para a população feminina. 69

Tabela 18: Taxa de chefia da população masculina ............................................ 72

Tabela 19: Taxa de chefia da população feminina .............................................. 72

Tabela 20: Projeções do número de domicílios ocupados .................................. 72

Tabela 21: Projeções de Unidades Consumidoras, Domicílios e Taxa de Atendimento 
"Nenhum homem será grande se desejar fazer tudo sozinho, ou receber todos os créditos por fazê-lo"

Andrew Carnegie 